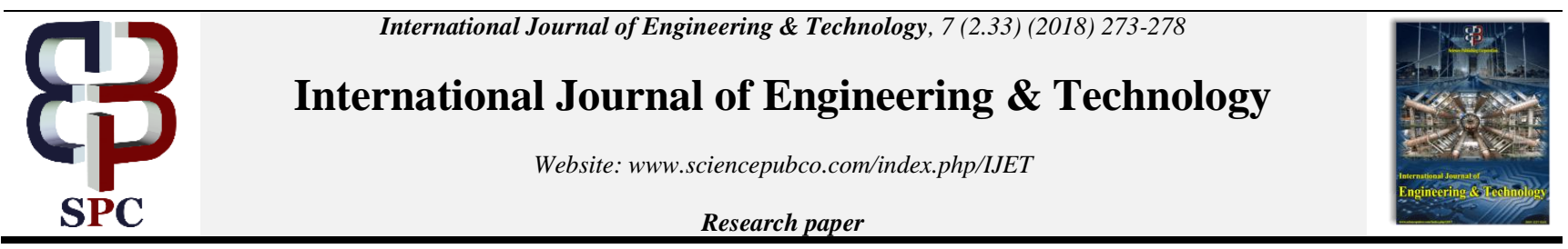

\title{
A survey on multimodal biometrics for human authentication
}

\author{
S. Arunarani ${ }^{1}$, R. Gobinath ${ }^{2 *}$ \\ ${ }^{1}$ Research Scholar, Department of Computer Science, VISTAS, Pallavaram, Chennai Assistant Professor, Department of Computer Sci- \\ ence, Prince Shri Venkates hwara Arts and Science College, Chennai \\ ${ }^{2}$ Department of Computer Science, VISTAS, Pallavaram, Chenna \\ *Corresponding author E-mail: sarunaarani@yahoo.co.in
}

\begin{abstract}
Authentication process identifies an individual to get an endorsed access by entering their login credentials. The inconvenience with this method is the user must remember the keywords, and the passwords can be predicted or if it is hard to guess it will be cracked through brute force. Due to this fault, this method is lack of integrity. Biometrics sample recognize a person based on his behavioral or physiological char-acteristics. Unimodal biometric systems have to resist with a different types of problems such as inconsistent data, intra-class variations, deceit attacks and high error rates. Multimodal biometrics implements secure authentication using various biometric traits. This survey gives us a wide scope for improving and enhancing the biometric applications. In this paper, we have explained multimodal biometrics to decrease the error rate and increase the security.
\end{abstract}

Keywords: Authentication; Biometrics; Multimodal Biometrics; Fusion; Score Level.

\section{Introduction}

In today's modern society, need for security extended far beyond personal culpability. Because of the importance of security concern among people, there is a need for new design to upgrade security which is more dependable and convincing. New prototype must allow device access only to legitimize users and deny access to illicit users. Biometrics is one such scheme which ensures a remarkable security to the system. The expression "Biometrics" originated from the Greek words, "bios" which means life and "metros" which means measure. Biometrics uses unique physiological and behavioral characteristics to identity an individual. Biometrics is designed to identify, interpret and measure an individual based on their physical and behavioral characteristics.

From the state or shape of the body, the physiological characteristics are determined. Fingerprint, face, ear, DNA, tongue, iris, palm print and hand geometry are examples of physiological characteristics. From the behavior of a person, the behavioral characteristics are determined. Keystroke dynamics, gait, signatures etc,. are examples of behavioral characteristics.

For biometric identification system, there are seven qualities a biometric should possess to provide high accuracy and efficiency. The qualities are singularity, universality, performance, stability, acceptability, quantifiability and circumvention.

\section{Biometric systems}

Biometric systems can be categorized into two types, (i) unimodal systems, (ii) multimodal systems.

\section{Unimodal biometric systems}

If the systems use single biometric traits of the individual for identification and verification then it is known as unimodal systems. Biometrics is used in many applications such as banking, online shopping, and immigration and electronic id card etc, Unimodal biometric identification may be appropriate for small scale of population but for large populations more problems emerge when there is single biometric. To overcome the single technology problem of unimodal, multimodal biometric systems are used.

Limitations of unimodal systems:

i) Non- universality: Occasionally people cannot render the single biometric trait because of their infirmity.

ii) Noise in perceived data: A user may be incorrectly rejected due to noise in the data which falsely matched with the templates in the database. For example, ear closed with hair, injury in the finger print are examples of noisy inputs.

iii) Lack of individuality: Sometimes, features extracted from different individuals can be quite similar. Example identical twins, father and son etc.,

iv) Lack of invariant representation: The user's template attained during enrollment are not identic with the data captured at the time of verification. 


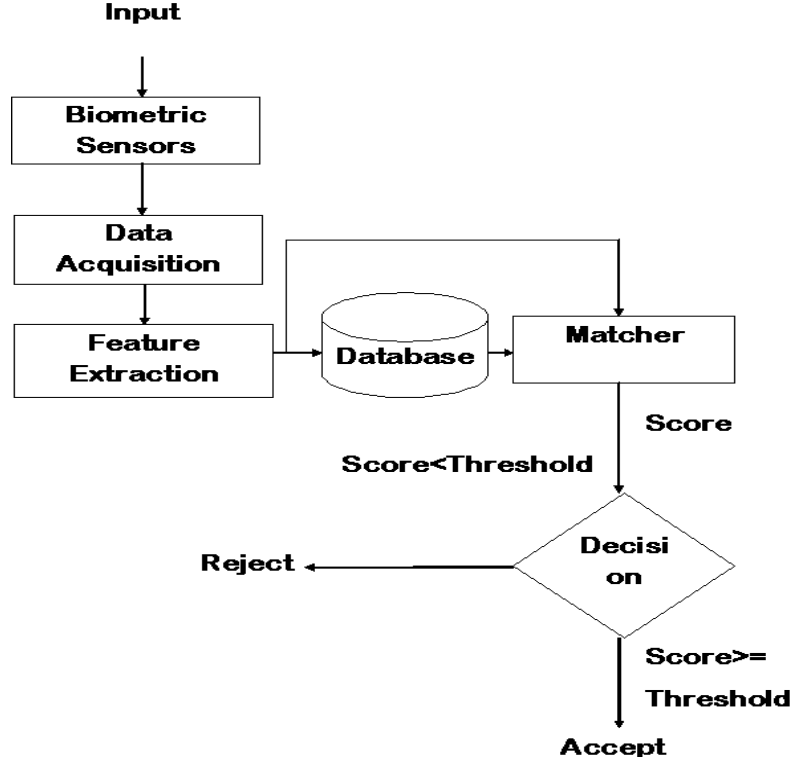

Fig 1: Simple Biometric System.

\section{Multimodal biometric system}

Multimodal biometric systems put to use more than one biometric trait for enrollment and identification.

False non-match and false match rates are reduced in immigration, network security and banking transactions by using multimodal biometric as a secondary means of enrolment and identifications. Multiple sensors are used for acquiring data in multimodal biometric system. This allows capturing multiple samples of a single biometric and / or samples of multiple biometrics trails called multimodal biometrics.

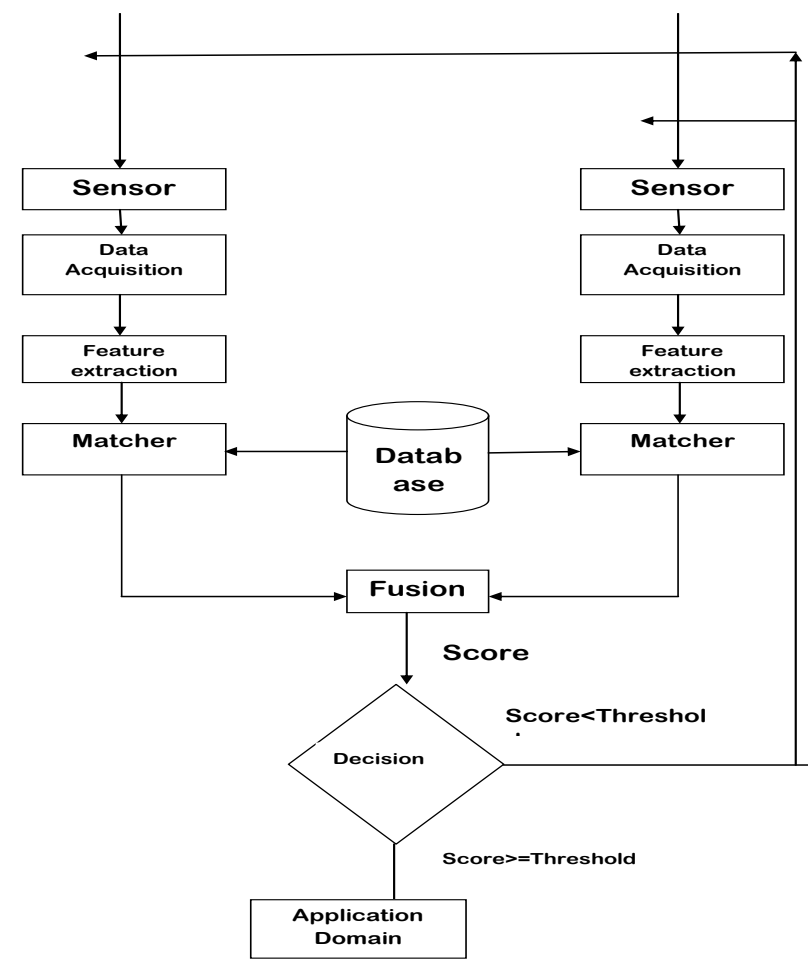

Fig. 2: Multimodal Biometric System.

Three different modes of operation in multimodal biometrics are as follows:

1) Serial mode: The outcome of one biometric trait is used to decrease the number of possible identities before the next trait is used.

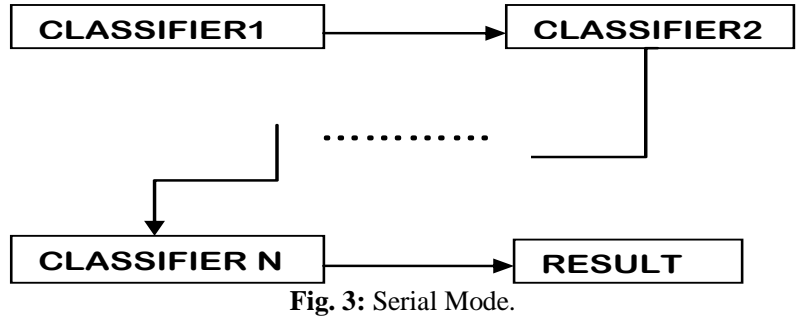

2) Parallel mode: Data from multiple biometric traits is used concurrently to enforce recognition.

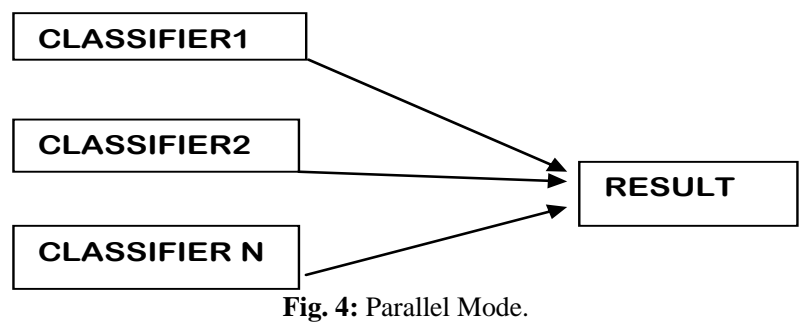

3) Hierarchical mode: Tree like structures is used to amalgamate individual classifiers.

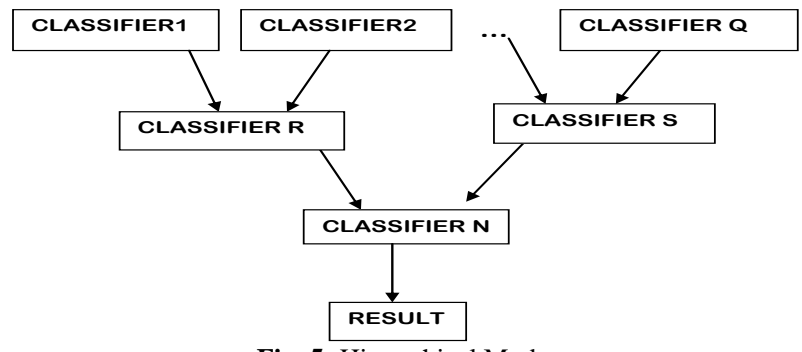

Fig. 5: Hierarchical Mode.

\section{Fusion approaches}

A multimodal biometric system need more than one decision channels, as there are two or more biometric traits are used. Biometric fusion is a mechanism which can combine the outcome from all the biometric modalities.

Fusion helps the implementation of biometric systems to improve fidelity, efficacy, validity, relevancy and universality. There are four levels of fusion in combining the biometric traits which can be used to elevate the reliability of the multimodal biometric system. They are

1) Sensor level fusion,

2) Feature level fusion,

3) Matching score level fusion

4) Decision level fusion:

1) Sensor level fusion: In this, biometric traits from different sensors are merged to form a combined trait for further process.

2) Feature level fusion: In this, feature vectors from different biometric channels are extracted individually from each biometric trait and then the vectors are merged to form a combined vector, which is used for further processing.

3) Matching Score level fusion: In this feature vectors from different biometric traits are processed and matching score of each and every trait is a calculated. Then, the matching scores are merged to produce a unified matching score which will be used for further categorization.

4) Decision level fusion: In this each and every biometric traits are classified separately as either accepts or rejects based on the extracted features. Then the absolute result is obtained by fusing the outputs from various traits. 


\section{Performance measurement}

In this section we describe how to evaluate the confidence of the accuracy indexes, for a biometric system.

The performance of a biometric system is measured using the following rates:

- FRR - False reject rate states the possibility of a genuine user being rejected by the system. It is a Type I error.

- FAR - False accept rate states the possibility of an unauthorized user being accepted by the system. It is a Type II error.

- Receiver (or relative) operating characteristic (ROC): ROC is a graphical plot drawn between the values of FAR and FRR for different variables.

- Crossover error rate(CER):

- CER is used to measure the accuracy of devices with various ROC curves. A device is said to be more accurate if CER rate is very low.

- Failure to enroll rate (FTE or FER):

- FER is the proportion of the raw data which vained the enrollment due to physical inability, or environmental disorders.

- Failure to capture rate (FTC):

- FCR transpire when the device fails to track down the biometric aspects which are conferred accurately.

Advantages of multimodal biometrics:

i) Multimodal biometric systems render high level of security, which is also more economical to run.

ii) Multimodal biometric systems provide high precision in identification of a person and FMR, FFMR rates are reduced.

iii) Multimodal biometric systems are not easily deceited as unimodal biometrics.

iv) Multimodal biometrics can reduce the deformity of data.

v) Multimodal biometrics provide significant boost in return on investment in various areas like Loss prevention.

Organization of the rest of the paper is as follows: section III discusses about the detailed review of the related work done in the field of multimodal biometric system. Section IV discusses about the optimum approach for person authentication. Section V discusses the conclusion and future work to be carried on to improve the performance of the system.

\section{Review of the related work}

In this section we summarize the techniques used by various authors, related to unimodal and multimodal biometric systems.
Multimodal biometrics has been proposed by Ross and Jain in 2003[3].Kames and Sule [4] presented identity management using Fingerprint and Iris. score level fusion is implemented. Mingxing Hea [5] proposed a multimodal biometric system based on fingerprint, face and finger vein. The performance of sum rule-based score level fusion and SVM based score level fusion are inspected in this paper. Imran [6] has proposed different normalization techniques on combining signature and fingerprint biometrics using feature level fusion to improve the performance.

Multimodal biometrics proves to be a very promising trend, in real-life biometric authentication applications.

Also, it is hard for an interrupter to deceit the multimodal biometric systems as multimodal biometrics is the fusion of various biometric data of a registered user.

A summary of work done by various authors in the field of unimodal and multimodal biometrics is rendered in the table 1 and table 2 .

The performance of biometric system can be improved by combining multiple traits and using score level fusion because of its ease to access facility and consolidating matching score. A table with work done by various authors in score level fusion is given in the table 3 .

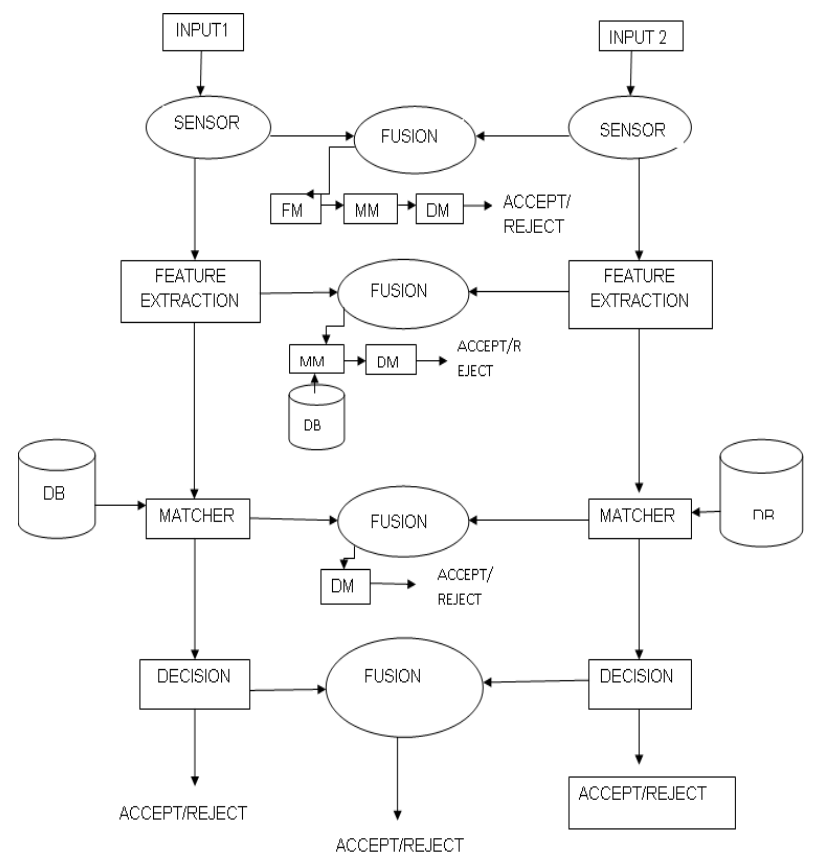

Fig6: Fusion Levels.

Table 1: Unimodal Biometrics

\begin{tabular}{|c|c|c|c|c|}
\hline Source (YEAR) & $\begin{array}{l}\text { Biometric } \\
\text { sources }\end{array}$ & Technique Adopted & $\begin{array}{l}\text { Performance of } \\
\text { Classification in percent- } \\
\text { age }\end{array}$ & Applications \\
\hline $\begin{array}{l}\text { Md. Mahbubur Rahman, Md. Rashedul } \\
\text { Islam, Nazmul Islam Bhuiyan, } \\
\text { Bulbul Ahmed, Md. Aminul Is- } \\
\text { lam('07)[25] }\end{array}$ & $\begin{array}{l}\text { EAR } \\
\text { Database }=100 \\
\text { samples }\end{array}$ & $\begin{array}{l}\text { Generalized Hugh Transform } \\
\text { (GHT) }\end{array}$ & Accuracy $=87 \%$ & Person identification \\
\hline Manisha RedhuDr.Balkishan('13)[26] & $\begin{array}{l}\text { Fingerprint } \\
\text { Database= } \\
\text { FVC2002 }\end{array}$ & Minutia Score matching method & $\begin{array}{l}\text { FAR }=30 \% \\
\text { FRR }=35 \% \\
\text { Accuracy }=65-70 \%\end{array}$ & Identity management \\
\hline $\begin{array}{l}\text { Sangram Bana and Dr. Davinder } \\
\text { Kaur9'[27] }\end{array}$ & Fingerprint & $\begin{array}{l}\text { Image Segmentation Minutiae } \\
\text { Matching }\end{array}$ & $\begin{array}{l}\text { FAR }=30 \% \\
\text { FRR }=35 \% \\
\text { Accuracy }=65-70 \%\end{array}$ & $\begin{array}{l}\text { Law enforcement and } \\
\text { immigration }\end{array}$ \\
\hline Gualberto Aguilar[28] & Fingerprint & $\begin{array}{l}\text { FFT (Fast Fourier Transform) } \\
\text { and Gabor filters }\end{array}$ & $\begin{array}{l}\text { RR of } 94.1 \%, \text { FAR of } \\
2.3 \% \text { FRR of } 3.6 \%\end{array}$ & Person Recognition \\
\hline Navneet Jindal, Vikas Kumar[29] & $\begin{array}{l}\text { Face } \\
\text { Database }=49 \\
\text { subjects }\end{array}$ & $\begin{array}{l}\text { PCA based eigenface, Artificial } \\
\text { Neural Networks with back } \\
\text { propagation process }\end{array}$ & Accuracy $=95.45 \%$ & Person Recognition \\
\hline $\begin{array}{l}\text { B S Venkatesh, S Palanivel and B } \\
\text { Yegnanarayana[30] }\end{array}$ & $\begin{array}{l}\text { Face } \\
\text { Database }=39 \\
\text { faces }\end{array}$ & $\begin{array}{l}\text { Eigen analysis of edginess, } \\
\text { Euclidean distance }\end{array}$ & Performance $=86 \%$ & $\begin{array}{l}\text { Face detection and } \\
\text { recognition in an image } \\
\text { sequence }\end{array}$ \\
\hline
\end{tabular}


Table 2: Multimodal Biometrics

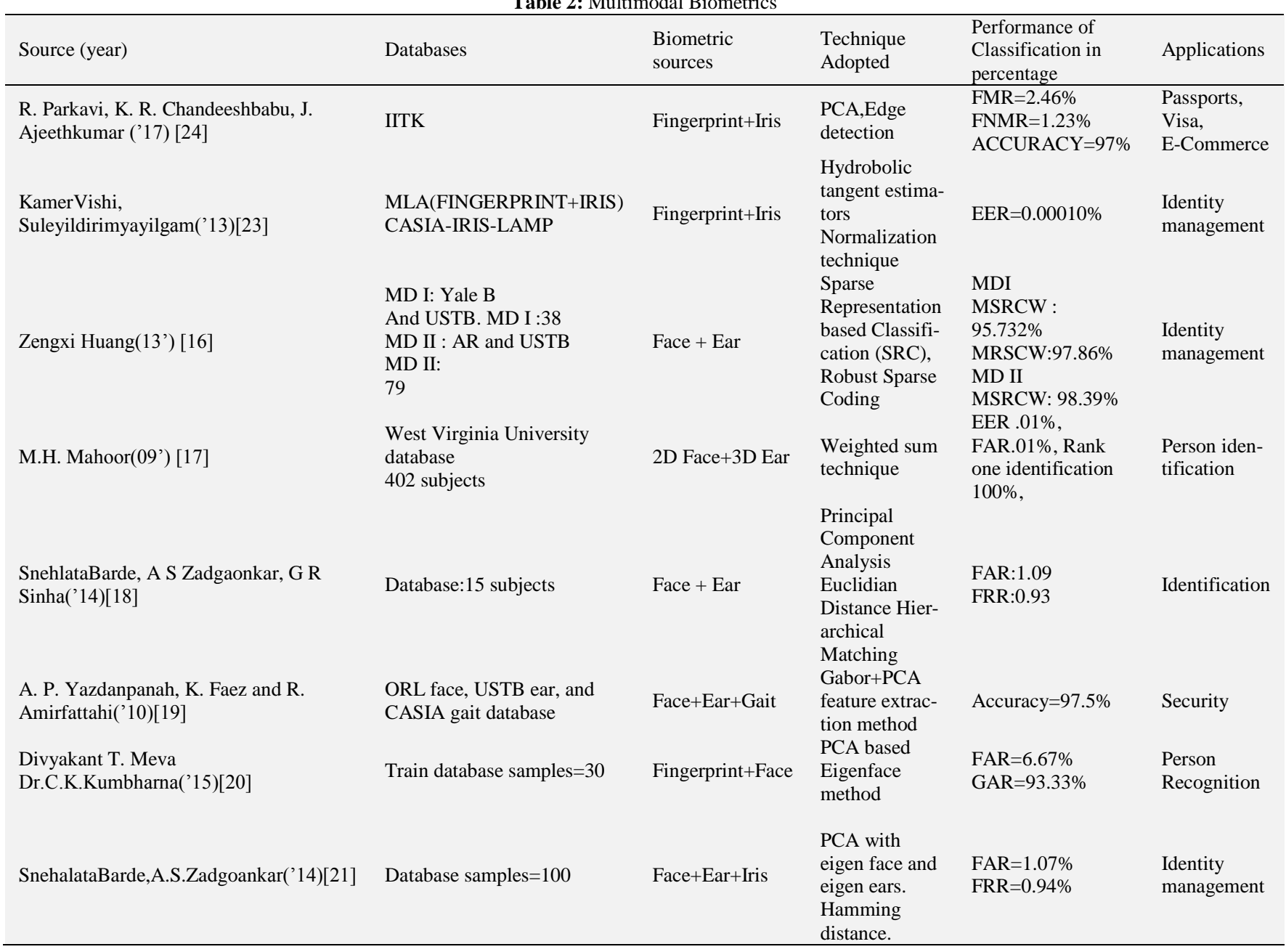

Table 3: Multimodal Using Score Level Fusion

\begin{tabular}{|c|c|c|c|c|}
\hline S.NO & $\begin{array}{l}\text { MODALITIES } \\
\text { TYPES }\end{array}$ & LEVEL OF FUSION & $\begin{array}{l}\text { FAR/FRR/ } \\
\text { GAR }\end{array}$ & REFERENCE \\
\hline 1. & Face and Voice & $\begin{array}{l}\text { Score level fusion. Gaussian mix- } \\
\text { ture. }\end{array}$ & $98.7 \%$ & $\begin{array}{l}\text { K.Nandakumar, Ychen, S.C .Dass and } \\
\text { A.K .Jain[2008] }\end{array}$ \\
\hline 2. & Fingerprint iris & $\begin{array}{l}\text { Score level fusion } \text {.Sum rule with } \\
\text { minmax. }\end{array}$ & $94.8 \%$ & K.Nandakumar, Ychen, A.K.Jain[2006] \\
\hline 3. & $\begin{array}{l}\text { Fingerprint (all fin- } \\
\text { gers) }\end{array}$ & Score level & $99.95 \%$ & B.Ulery, A.hicklin , C.Watson,[2006] \\
\hline 4. & $\begin{array}{l}\text { Fingerprint face, } \\
\text { fingervein }\end{array}$ & Score level & $99.8 \%$ & Shi - Jinn Horng, Kevin OctavisSentosal[2009] \\
\hline 5. & $2 \mathrm{D} \& 3 \mathrm{D}$ face & Score level & $99.4 \%$ & $\begin{array}{l}\text { TaherKhadhraoui, Faouzi Ben Zarti, Hamid Amiri } \\
\text { ICIS IEEE - } 2014\end{array}$ \\
\hline 6. & Fingerprint iris & Score level & $94.17 \%$ & KamelAizi, Mohamed ouslim, Ahmed sabri[2015] \\
\hline
\end{tabular}

\section{Optimum approach for person authentica- tion}

The choice of the biometric traits plays a vital role in estimating the performance of the biometric systems. Each biometric trait has its own strength and weakness. Several multimodal biometric systems were developed by combining different biometric modalities. The aim of multi biometrics is to improve quality of recognition over an individual method by combining the results of multiple features, sensors or algorithms. We need to create a multimodal system which shows better performance, less complexity, lower cost and easily obtainable.

1) Fingerprint:

Fingerprints are used to identify person from the unique pattern of whorls and lines on it. It is obtained electronically by scanning the finger image and it is stored for further processing. No two human being in the world have been found to have identical fingerlprints, even twins have different fingerprints.
Fingerprints are more exclusive than the genetic traits. Finger print identification is one of the oldest and widely known biometrics. it have been used for over a century, because of its flexibility and exclusiveness. Digital image of a fingerprint is compiled using different types of sensors.some of them are optical,thermal,capacitive sensors etc., Among this, optical sensors are used commonly. 


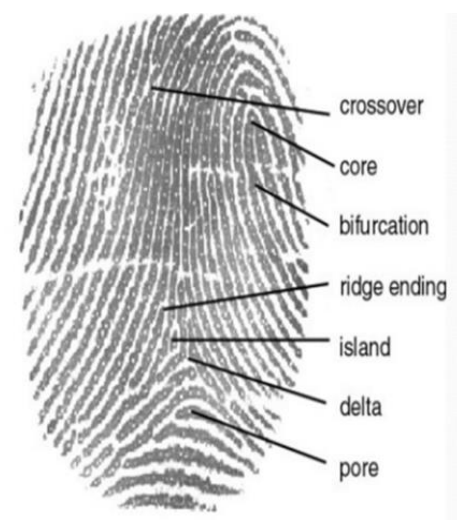

2) EAR

The human ear is one of the more multifaceted organs of the human body. The visible part of the ear is called Pinna, which acts as a funnel to amplify the sound. The shape of the outer ear, pinna is unique for every human being. Because of its uniqueness and constancy, it is used for human identification. The growth of ear from 5 months to 8 years is meager and after that it is stable till 70 years. Due to its uniqueness and stability, ear is used as a promising biometric. Generally, digital image of the ear is obtained alike face images, using digital camera. It doesn't require any scanning of a trait over a identification device. It can be operated even when the human is moving. So, ear can be well chosen as a biometric.

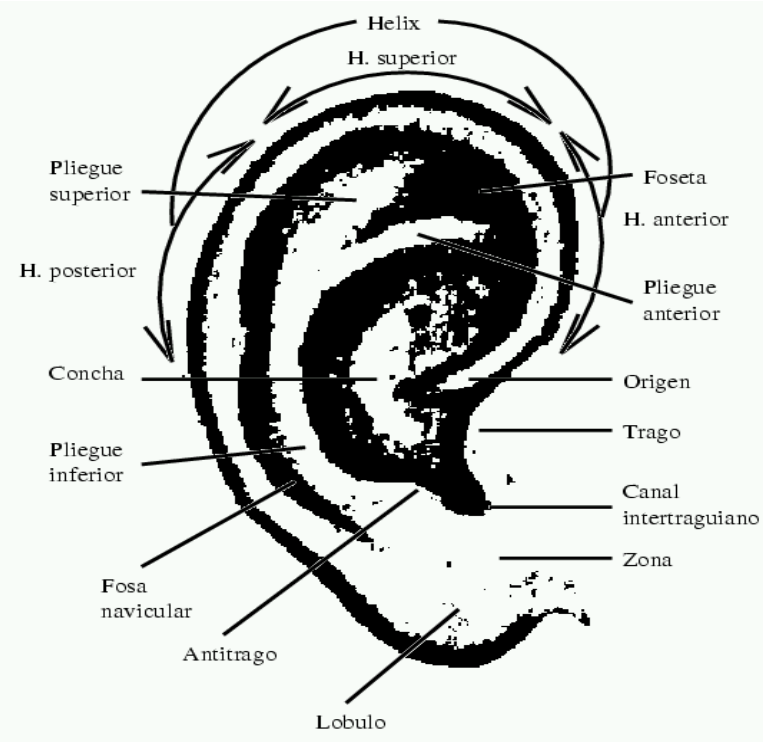

3) Face

Human mind is more accurate in identifying the person based on their face. Similarly, computer is programmed to identify the person at a glimpse of images of the human being for a few milliseconds to accurately correlate the face. The face starts from the hair lane to chin and from ear to ear. The face has nose, mouth and eyes. Identifying a person is based on the position of nose, mouth and eye in the face.

In holistic approach, the complete face region is taken into account as input data into face catching system. One of the best example of holistic methods are Eigenfaces [8] (most widely used method for face recognition), Principal Component Analysis, Linear Discriminant Analysis [7] and independent component analysis etc.

In feature- based method, the local features and their locations are delivered to a classifier for identification. The main applications of facial recognition lies in online payments, criminal identifications, and security and accessing by integrating the facial biometrics with physical devices and objects.
In Australia, Brisbane airport succeeded facial recognition trial for check-in, which saves $70 \%$ of passenger processing time. In japan, facial recognition technique is used for border security.
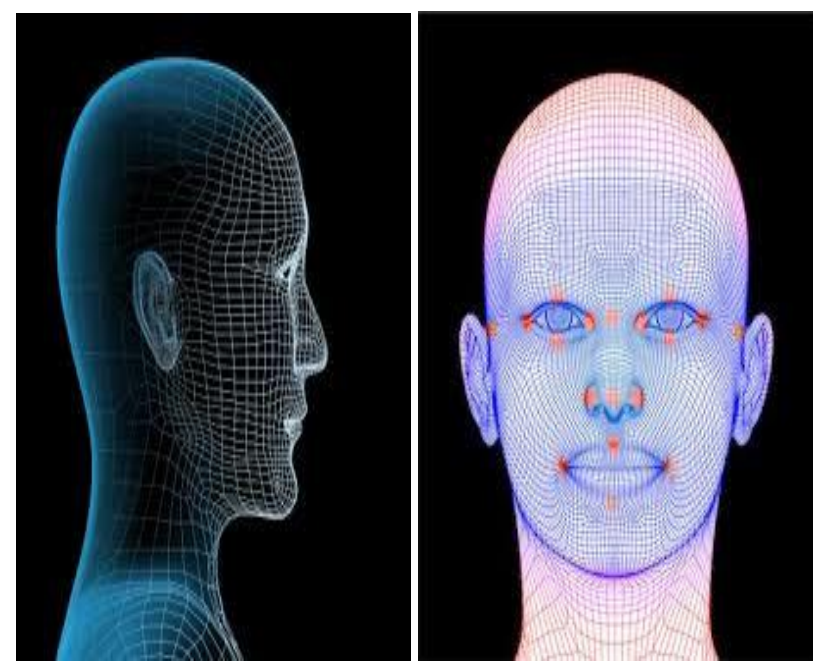

The user's facial image is captured and is processed and matched against the related template.

The fused features of face, ear and fingerprint are processed and matched against the related template. If the score is more than the threshold, the user is accepted as a genuine user and else it is declared as imposter. This proposed system reduce FAR and computation time for person identification.

\section{Conclusion}

Multimodal biometric systems are discussed here. Fusion of ear and fingerprint and face is proposed. Our interest is to investigate how well the three modalities can mutually benefit from each other to result into a fortified system with high recognition capability despite the spoof attempts. Future work will focus upon the development of an authentication prototype with higher level of recognition performance that can be achieved.

\section{References}

[1] A.K.Jain, Arun Ross, S.Prabakar, "An Introduction to bometric recognition" IEEE Trans. Circuits and Systems for Video Technology Special issue on image and video based biometrics, vol 14, PP 4-20, January 2004.

[2] S.C.Joshi, Dr.Abhay Kumar, "Design of multimodal biometrics syatem based on feature level fusion" IEEE.

[3] A.Ross and A.K. Jain "Information fusion in biometrics, Pattern recognition letters vol -24, 2003 PP.2115-2125.

[4] Kames Vishi, SuleYildiriumYayilgen. "Multimodal Biometrics Authentication using fingerprint and iris" IEEE 2013 PP.334-341.

[5] MingxingHea, Shi-Jinnhorng, "Performance evaluation of score level fusion in multimodal biometric systems" Volume 43 Issue 5 (2010) 1789 - 1800 .

[6] Mohammad Imran, Hementha, "Accurate Person recognition on combining signature and fingerprint", International Journal of Machine Intelligence ISSN: 0975-2927 Volume 3, Issue \$, 2011 PP277-281.

[7] Shi - Jinn Horng, Kevin OctavisSentosal, "An Improved Score level fusion in Multimodal Biometric systems" IEEE ISSN 7695 -3914 2009.

[8] "Multimodal Hybrid Face recognition based on score level fusion using RVM". TaherKhadhraoui, Faouzi Ben Zarti, Hamid Amiri ICIS IEEE - 2014.

[9] KamelAizi, Mohamed ouslim, Ahmed sabri"Remote multimodal Biometric identification based on the fusion of the iris and the fingerprint".IEEE 2015 ISSN: 4673-6673.

[10] K.Nandakumar, Ychen, S.C .Dass and A.K .Jain," Likelihood Ratio - based Biometric Score fusion".IEEE Transaction on Pattern Analysis, Vol 30 Feb 2008. PP -342-347. 
[11] K.Nandakumar Ychen, A.K.Jain "Quality - based score level fusion in multibiometric systems" ICPR, Vol 4, 2006 PP.473- 476.

[12] [12].B.Ulery, A.hicklin, C.Watson,'Studies of Biometric fusion executive Summary" NISTIR 7346, Sep 2006.

[13] Shi-Jinn Horng, Kevin,"An Improvrd score level fusion in multimodal biometric systems."

[14] MayersMahadevSawant, Yogesh NagarGeogeetal "Keystroke dynamics: review paper "ISARCCE, Vol 2 Oct 2013.

[15] P.Gnanasivam Dr.S.Multan, "Ear and fingerprint Biometrics for Personal Identification" ICSCCN 2011.

[16] Zengxi Huang, Yiguang Liu, Chunguang Li, Menglong Yang and Liping Chen, "A robust face and ear based multimodal biometric system using sparse representation",Pattern Recognition, 2013, Vol. 46, No. 8, pp.2156-2168.

[17] Mohammad H. Mahoor Steven Cadavid, and Mohamed AbdelMottaleb, "Multi-modal Ear and Face Modeling and Recognition", Proc. IEEE 16th International Conference on Image Processing, 2009, pp. 4137-4140.

[18] SnehlataBarde, AN S Zadgaonkar, G R Sinha,"PCA based Multimodal Biometrics using Ear and Face Modalities", IJITCS, vol.6, no.5, pp.43-49, 2014.

[19] A. P. Yazdanpanah, K. Faez and R. Amirfattahi, "Multimodal biometric system using face, ear and gait biometrics," 10th International Conference on Information Science, Signal Processing and their Applications (ISSPA 2010), Kuala Lumpur, 2010, pp. 251-254.

[20] Divyakant T. Meva, Dr.C.K.Kumbharna, 'Design and evaluation of multimodal biometric system with fingerprint and face recognition"IJSRP VOL5 ISSUE 4 APRIL 2015 ISSN 2250-3153

[21] SnehalataBarde, A.S.Zadgoankar, "Multimodal Biometric using face,ear and iris modalities" International Journal of Computer Applications (0975-887)2014

[22] KamelAizi,MohamedOuslim,AhmedSabri, Electronics Department USTO,Algeria "Remote Multimodal Biometric identification Based on the fusion of the iris and the fingerprint",2015

[23] KamerVishi,Suleyildirimyayilgam, "GjovikCollege,Norway,'Multimodal Biometric Authentication using fingerprint and iris" International conference on Intelligent Information Hiding,2013

[24] R.Parkavi,K.R.Chandeeshbabu,J.Ajeethkumar, "Thiagarajar University, "Multimodal Biometrics for User Authentication", ISCO 2017

[25] Md. Mahbubur Rahman, Md. Rashedul Islam, Nazmul Islam Bhuiyan,Bulbul Ahmed, Md. Aminul IslamComputer Science and Engineering Discipline, Khulna University," Person Identification Using Ear Biometrics" International Journal of The Computer, the Internet and Management Vol. 15\#2 (May - August 2007) pp 1 -8.

[26] Manisha Redhu 2 Dr.Balkishan, "Fingerprint Recognition Using Minutiae Extractar", International Journal of Engineering Research and Applications (IJERA) ISSN: 2248-9622 www.ijera.com Vol. 3, Issue 4, Jul-Aug 2013,pp .2488-2497

[27] Sangram Bana and Dr. Davinder Kaur , "; Fingerprint Recognition using Image Segmentation" / (IJAEST) INTERNATIONAL JOURNAL OF ADVANCED ENGINEERING SCIENCES AND TECHNOLOGIES Vol No. 5, Issue No. 1,012 - 023

[28] G. Aguilar, G. Sánchez, K. Scano, M. Salinas, M. Nakano, and H. Perez, -Fingerprint recognition, Second International Conference on Internet Monitoring and Protection, IEEE, 2007.

[29] Navneet Jindal , Vikas Kumar," Enhanced Face Recognition Algorithm using PCA with Artificial Neural Networks", Volume 3, Issue 6, June 2013 ISSN: 2277 128X International Journal of Advanced Research in Computer Science and Software Engineering

[30] B S Venkatesh, S Palanivel and B Yegnanarayana Department of Computer Science and Engineering. Indian Institute of Technology, Madras," Face Detection and Recognition in an Image Sequence using Eigenedginess"

[31] Gopal Singh Tandel Proceedings of ICIRT 2012,” Ear Recognition" 2012 pg. 171-173

[32] Bolle, Ruud M., Jonathan Connell, SharathPankanti, Nalini K. Ratha, and Andrew W. Senior. Guide to biometrics. Springer Science \& Business Media, 2013.

[33] Jain, Anil K., KarthikNandakumar, and Abhishek Nagar. "Biometric template security." EURASIP Journal on Advances in Signal Processing 2008 (2008): 113. 